\title{
OnTask: Delivering Data-Informed, Personalized Learning Support Actions
}

\author{
Abelardo Pardo, ${ }^{1}$ Kathryn Bartimote-Aufflick, ${ }^{2}$ Simon Buckingham Shum, ${ }^{3}$ Shane Dawson, ${ }^{4}$ Jing \\ Gao, ${ }^{5}$ Dragan Gašević, ${ }^{6}$ Steve Leichtweis, ${ }^{7}$ Danny Liu, ${ }^{8}$ Roberto Martínez-Maldonado, ${ }^{9}$ Negin \\ Mirriahi, ${ }^{10}$ Adon Christian Michael Moskal, ${ }^{11}$ Jurgen Schulte, ${ }^{12}$ George Siemens, ${ }^{13}$ Lorenzo \\ Vigentini ${ }^{14}$
}

\begin{abstract}
The learning analytics community has matured significantly over the past few years as a middle space where technology and pedagogy combine to support learning experiences. To continue to grow and connect these perspectives, research needs to move beyond the level of basic support actions. This means exploring the use of data to prove richer forms of actions, such as personalized feedback, or hybrid approaches where instructors interpret the outputs of algorithms and select an appropriate course of action. This paper proposes the following three contributions to connect data extracted from the learning experience with such personalized student support actions: 1) a student-instructor centred conceptual model connecting a representation of the student information with a basic set of rules created by instructors to deploy Personalized Learning Support Actions (PLSAs); 2) a software architecture based on this model with six categories of functional blocks to deploy the PLSAs; and 3) a description of the implementation of this architecture as an open-source platform to promote the adoption and exploration of this area.

\section{Notes for Practice}

- The report draws on research findings related to the effect of personalized feedback on student satisfaction and academic performance (Pardo, Jovanović, Dawson, Gašević, \& Mirriahi, 2018).

- The main contribution is the description of the design and implementation of an open source platform for researchers and practitioners to connect data with personalized learning support actions.

- The area of learning analytics needs tools such as the one described in this document to serve as a vehicle to exchange insights among researchers and practitioners.

- This is an example of the note for practice and research
\end{abstract}

\section{Keywords}

Learning analytics, feedback, personalization, open source, student suppoer.

Submitted: 22.03.2018 - Accepted: 26.07.2018 - Published: 11.12.2018

Corresponding author ${ }^{1}$ Email: abelardo.pardo@unisa.edu.au Address: Campus Central - City West University of South Australia GPO Box 2471 Adelaide SA 5001, ORCID ID: 0000-0002-6857-0582

${ }^{2}$ Email:kathryn.bartimote@sydney.edu.au Address: The University of Sydney NSW 2006, Australia, ORCID ID: 0000-0002-6989-3754

${ }^{3}$ Email: Simon.BuckinghamShum@uts.edu.au Address: University of Technology Sydney, PO Box 123, Broadway NSW 2007, Australia, ORCID: ID 0000-0002-6334-7429

${ }^{4}$ Email:shane.dawson@unisa.edu.au Address: Campus Central - City West University of South Australia GPO Box 2471 Adelaide SA 5001, ORCID ID: 0000-0003-2435-2193

${ }^{5}$ Email:jing.gao@unisa.edu.au Address: Campus Central - City West University of South Australia GPO Box 2471 Adelaide SA 5001

${ }^{6}$ Email:dragan.gasevic@monash.edu.au Address: Monash University, Victoria 3800, Australia, ORCID: ID: 0000-0001-9265-1908

7Email: s.leichtweis@auckland.ac.nz Address: The University of Auckland, Private Bag 92019, Victoria Street West, Auckland 1142, New Zealand, ORCID ID: 0000-0002-4381-2901

${ }^{8}$ Email:danny.liu@sydney.edu.au Address: The University of Sydney NSW 2006, Australia

${ }^{9}$ Email:roberto.martinez-maldonado@uts.edu.au Address: University of Technology Sydney, PO Box 123, Broadway NSW 2007, Australia, ORCID ID: 0000-0002-8375-1816

${ }^{10}$ Email:negin.mirriahi@unisa.edu.au Address: Campus Central - City West University of South Australia GPO Box 2471 Adelaide SA 5001, ORCID ID: 0000-0003-4139-3149

${ }^{11}$ Email: Adon.Moskal@op.ac.nz Address: Corner Erris \& Ray Streets, PO Box 16, Cromwell, New Zealand 9342, ORCID: ID: 0000-0003-40892130

${ }^{12}$ Email:jurgen.schulte@uts.edu.au Address: University of Technology Sydney, PO Box 123, Broadway NSW 2007, Australia, ORCID ID: 00000001-8845-8099

13Email:gsiemens@gmail.com Address: Campus Central - City West University of South Australia GPO Box 2471 Adelaide SA 5001

${ }^{14}$ Email:I.vigentini@unsw.edu.au Address: UNSW Sydney, NSW 2052, Australia, ORCID: ID 0000-0002-8436-7621 


\section{Introduction}

Learning analytics research has matured significantly over the past few years. In particular, the field has made significant advances in areas surrounding learner behaviour modelling (Liu, Patel, \& Koedinger, 2016; Pelánek, Rihák, \& Papoušek, 2016) and text analytics (Crossley, Kyle, \& McNamara, 2015; Buckingham Shum et al., 2016). Despite these advances, questions of how analytics are best deployed and applied in learning and teaching practice (Wise, 2014; Wise, Vytasek, Hausknecht, \& Zhao, 2016) continue to be underrepresented in the literature. In essence, the field has well progressed in the more technical aspects of learning analytics research yet the application of data to improve learning has been at best sporadic.

To further advance learning analytics, the field must move beyond the simple provision of information as one-way feedback to students (e.g., passive learning dashboards) based on a single (or limited range) of data sources. Learners require richer forms of information and more effective support, such as feedback processes that consider different dimensions of the learning experience, personalized using multiple data sources. We argue that the creation of this feedback process should be informed by data sources, but not driven solely by them. Recent reports in the area of artificial intelligence in education have also pointed to the need to move beyond algorithmic solutions that replicate human intelligence in relatively restrictive settings (Ma, Adesope, Nesbit, \& Liu, 2014; Niculescu, 2016) and consider hybrid approaches to learning analytics where instructors interpret the results produced by algorithms and select appropriate actions (Baker, 2016; Bull \& Kay, 2016). Although areas such as expert systems or decision support systems have explored in depth how to support humans in their decision-making processes, their presence in educational settings is scarce or restricted to highly specific topics. When applied in educational contexts, adaptive systems require sophisticated knowledge domain and resource-intensive user models, often having a low level of adoption among instructors (Nye, 2014).

In response to some of the above limitations, we have seen in recent years the emergence of systems that draw from multiple data sources and operate on relatively simple rule-based logic (when compared to, for example, intelligent tutoring systems that require a comprehensive representation of the domain of knowledge and the state of the learner) to improve the feedback given to learners (Echeverria et al., 2018). These systems generally require far less knowledge engineering effort and have the added benefits of letting instructors compose personalized feedback messages to students specifically for their courses, drawing on data already held by their institution. This paper seeks to bridge the current disconnect between the provision of algorithmic solutions and instructor-learner practice by distilling the key elements that appear to make such systems successful. In doing this, the paper presents a human-centred model, and its associated toolset, that allows the provision of Personalized Learning Support Actions (henceforth, PLSAs) at scale based on multiple student data. To promote the adoption of the model, the paper presents an approach that starts from an entity similar to a spreadsheet, established as easy to use in increasing productivity of professionals in different areas (Scaffidi, Shaw, \& Myers, 2005), and combines it with a set of basic rules to personalize the actions for each student.

The openness of processes, algorithms, and technology in learning analytics solutions is crucial in fostering innovation and widespread adoption in higher education institutions (Siemens et al., 2011). Some initiatives have built elements of open platforms; however, they are mainly focused on the technical (data and analysis) aspects instead of teaching and learning processes (Jayaprakash, Moody, Eitel, Regan, \& Baron, 2014). The goal of an open learning analytics platform (OLA) was proposed in the past, but still remains at the conceptual level (Chatti, Muslim, \& Schroeder, 2017). The implementation and availability of open source learning analytics tools is also at its initial stage. Initiatives such as xAPI (Experience API, 2016) and Caliper (IMS Consortium, 2016) focus on technological aspects of how to capture, encode, and manage events, but do not propose any specific solution for the deployment of support actions. The paper proposes three contributions: 1) a studentinstructor centred conceptual model connecting a representation of the student information with a basic set of rules created by instructors to deploy PLSAs; 2) a software architecture based on this model with six functional categories; and 3) a description of the implementation of this architecture as an open-source.

\section{Background}

The field of learning analytics is continuing to progress from initial scenarios of using data to predict which students are at risk of withdrawal or academic failure, to more generic scenarios in which data are used to decide how best to improve the learning experience and academic outcomes of all students (Sclater, Peasgood, \& Mullan, 2016; Shacklock, 2016). However, much of the effort in this trajectory has been devoted to aspects such as monitoring (Romero Zaldívar, Pardo, Burgos, \& Delgado Kloos, 2012), predicting (Romero \& Ventura, 2013), visualizing student behaviour (Verbert, Duval, Klerkx, Govaerts, \& Santos, 2013), and supporting students at a holistic level within an institution (Shamah \& Ohlsen, 2013). The phrase closing the loop (Clow, 2012) has been used to describe the need to connect actions (or interventions) back to the learners. Yet, this connection has not been sufficiently explored. For instance, while analytic dashboards are powerful tools to convey information to students, measuring the impact of such dashboards is challenging and may require longitudinal studies (Verbert et al., 2013). Additionally, students may not be adequately prepared to interpret the dashboard information properly, meaning the provision of analytics could have no effect, or even have a negative effect on their academic performance (Corrin \& de Barba, 2015; Lonn, Aguilar, \& Teasley, 2015).

ISSN 1929-7750 (online). The Journal of Learning Analytics works under a Creative Commons License, Attribution - NonCommercial-NoDerivs 3.0 Unported (CC BY-NC-ND 3.0) 
Other systems have adopted a holistic approach to student support at the level of the institution. Platforms such as the Student Success Plan (Shamah \& Ohlsen, 2013) help institutions to manage general advising and coaching actions. Despite the work that has been undertaken to date, there remains a strong need to investigate how analytics are best translated into viable and productive learner actions (Wise et al., 2016). In this paper, we propose a system to provide more effective student support within the context of a course.

Feedback is a key element by which students and instructors engage in a dialogue with the aim of improving the learning experience (Nicol, 2010). While feedback and its deployment in learning scenarios have been the focus of intensive research (Evans, 2013; Hattie \& Timperley, 2007; Nicol, Thomson, \& Breslin, 2013 for more detailed reviews), very few studies have explored how it can be adequately provisioned to students as a support action informed by learning analytics. Hattie and Timperley (2007) proposed four levels at which feedback can be provided to students: 1) the task level, 2) the learning process level (at which tasks are understood), 3) the self-regulation level, and 4) the level of self (including affect and personal evaluation). Feedback was most effective at the learning process and self-regulation levels that require non-trivial sensemaking steps performed by instructors but it is extremely challenging to scale to large numbers of students.

The area of Educational Data Mining (EDM) has also dealt with the provision of feedback (Hegazi \& Abugroon, 2016) from the point of view of student and predictive modelling research. The focus is on producing feedback messages that adapt to student needs by facilitating improvements, reinforcing academic performance, or dissuading certain undesired behaviours (Romero \& Ventura, 2013). Most of the contributions approach feedback at the task level, providing corrective feedback or the capacity to visualize data sets just-in-time for instructors to decide which actions are most appropriate (Lewkow, Zimmerman, Riedesel, \& Essa, 2015).

The need to focus on actionable knowledge extends also to tool development. In a recent article, Gašević, Dawson, and Siemens (2015) pointed out that tool development in learning analytics is not taking into account instructional strategies, especially those around the provision of student feedback. The model, architecture, and implementation described in this paper focus on the provision of personalized student support actions at the level of a course. We propose a system in which: 1) students are explicitly acknowledged as the ultimate beneficiaries of data-driven support actions; and 2) instructors are essential for connecting the data, the learning environment, and the students through the deployment of PLSAs. We use feedback as the target concept to establish an explicit connection between the data available in a learning environment and the decisions made by an instructor to provide personalized support to students. This relation is usually assumed but not explicitly represented. In this paper, we describe OnTask, a tool to express the instructor insight gained while exploring data and deploying PLSAs for students at scale.

\section{Related Work: Connecting Data with Student Support Actions}

Several research studies have identified the need for a systemic approach to the problem proposed in this paper. For example, Brooks, Greer, and Gutwin (2014) described three case studies whereby instructors used data captured from a learning environment to gain insight about their teaching practice. In the first case, instructors were provided with visualizations that prompted them to make adjustments in their pedagogical practice. No explicit action is mentioned to support the students. In the second case study, data mining techniques were used to showcase the relation between events in the lecture capture system and educational outcomes. The resulting model was intended to be used by the instructor to alert students surrounding a lack of engagement with the learning materials. No specific details were provided on how to capture this action and trace its impact. The third case study provided a concrete intervention to support students. The opinions of six human subjects in a laboratory about the relevance of indices placed on recorded lecture videos were collected and analyzed. The results allowed educational technologists to personalize navigational support through the system. These interventions considered the insight of instructors as an important part of the process to detect intervention; however, they lacked a systematic framework in which actions connect with insights. These three studies highlight the importance of including sensemaking and the deployment of actions as part of the overall problem. As presented, the support actions would be difficult to deploy, either to large numbers of students or in other courses with similar learning scenarios. The system presented in this paper proposes a formalism for instructors to state explicitly the relation between data and actions so that they can be scaled to reach large numbers of students frequently and in multiple learning scenarios.

The $E^{2}$ Coach platform (Wright, McKay, Hershock, Miller, \& Tritz, 2014) developed at the University of Michigan combined detailed data about the students with the advice of instructors and students and encoded such knowledge in a logic framework to deliver coaching messages to large student cohorts. The information was provided in a web page with content personalized for each student. The process to capture the expertise of instructors and students was done through focus groups and interviews and was later encoded in the system. The system captured expert knowledge but through a time-consuming 


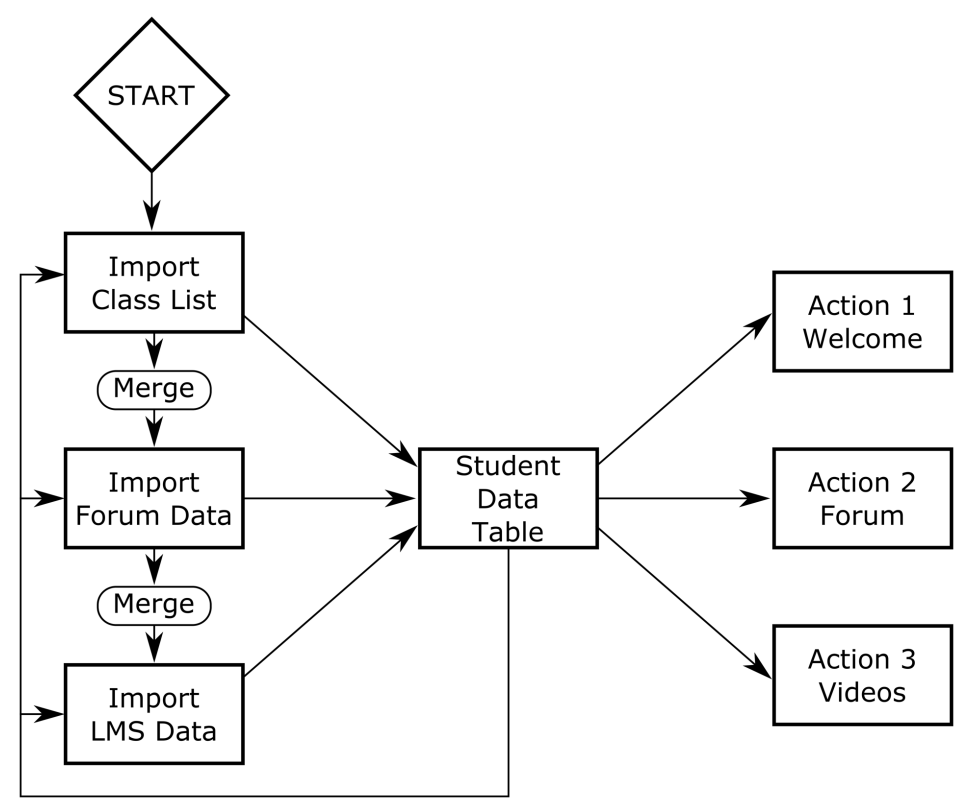

Figure 1. Example of an OnTask workflow.

process (i.e., interviews) that was highly sensitive to its context. We propose an approach to capture these insights through a simple formalism so that it can be easily scaled and shared across scenarios.

A more holistic approach to student support has been addressed by the Student Success Plan (Shamah \& Ohlsen, 2013) developed by Unicon, a provider of IT services in the US, and adopted by various institutions. The system offers a comprehensive, yet generic, management process to handle student support actions ranging from coaching, academic advice, study techniques suggestions, course advise, etc. Our approach aims to capture the connection between data, expert insight, and actions from the point of view of the instructor, localized to a specific learning experience. This focus allows more specific interventions, as instructors are in a unique position to provide more valuable suggestions to improve study habits since they are directly connected with elements of the instructional design.

The Student Relationship Engagement System (SRES) developed at The University of Sydney (Liu, Bartimote-Aufflick, Pardo, \& Bridgeman, 2017) underpins much of the work presented in this paper. The SRES approached the problem from the point of view of empowering instructors to capture the provision of personalized data-supported feedback to students through the definition of conditions. Students received personalized messages with information and suggestions created depending on the data obtained from their engagement in the learning environment. Instructors wrote conditions to select the appropriate parts of the message, personalized for each student. This approach relied on the instructor's knowledge of both the learning design and their students, and the capacity of the instructor to provide highly specific feedback. Our proposed conceptual model helps to abstract this paradigm to accommodate a richer set of data sources and the provision of generic support actions (other than messages).

A similar scenario was observed in a first year Engineering unit at The University of Sydney. Students were given weekly tasks as part of an active and blended learning environment together with a dashboard updated in real time with an indication of their engagement (Kahn \& Pardo, 2016). The dashboard also contained suggestions on how to engage with the course tasks, and the text changed in real time based on the data captured from the learning environment. Although the provision of feedback had a positive impact on student perception, the connection between data and the suggestions was done in an ad-hoc fashion unsuitable for widespread adoption beyond the course. A later study showed that complementing the dashboard with databased personalized messages produced a significant increase in student satisfaction with feedback and a slight increase in academic performance (Pardo et al., 2018).

All the initiatives described in this section try to establish a connection between data, the learning environment, expert knowledge, and actions to support students. However, they all highlight the need for an approach that considers these elements together under the lens of supporting students in their learning. This paper proposes the definition of a conceptual model that attempts the following: 1) abstracts the details of each of the solutions and provides a common structure to represent the data; 2) captures in a lightweight formalism the instructor and learning designer expertise with respect to the underlying instructional design; and 3) establishes a formal connection with PLSAs. 


\section{Conceptual Model and Stakeholders}

The proposed conceptual model adopts an intelligence augmentation approach (Baker, 2016) combining algorithmic techniques that include data capturing and pre-processing, with human decisions represented by the design of the PLSAs. Figure 1 illustrates an example of the workflow envisioned for the model. Users import an initial class list with basic student information into the student data table. Additional data sources are then merged to populate additional columns (or attributes) for the student data table. Once the data table has been populated, three examples of actions are shown. The first one includes a welcome message personalized based on some table attribute. For example, the message may refer to the relevance of the course within the overall program if there are students from different programs. The second action provides ideas and suggestions about how to participate in the discussion forum in the course (assuming there is one.) This message is personalized based on the data about student engagement with the forum. A similar scenario is assumed for action 3 in which the message is personalized based on the student engagement indicators with the course videos.

\subsection{The Conceptual Model}

Figure 2 illustrates the proposed conceptual model. Its structure has been influenced by Diana Laurillard's Conversational Framework (Laurillard, 2013) because its primary purpose is to support the communication cycle between students and instructors through the deployment of PLSAs. This communication occurs at certain points in time during the learning experience through a variety of channels (e.g., messaging platforms, changes in the learning environment, and resources) and is informed by the data collected in the student data table and the instructor insights. The model has six key elements (boxes 1-6 in Figure 2) described in detail below.

\subsubsection{Box 1: Data Warehouse}

The cycle commences when students interact with activities in the learning environment supporting the achievement of a set of intended learning outcomes. If these interactions occur in a technology-mediated environment (typically a Learning Management System [LMS]), the system captures detailed sequences of events and stores them in the entity called Data Warehouse (box 1 in the model - Figure 1). Examples of the events captured include solutions provided by students to problems related to their required course readings, video annotations, discussions in a forum, content of written assignments, etc.

\subsubsection{Box 2: Other Data Sources}

Not all events in the learners' experience take place within the LMS. The entity named Other Data Sources represents all potential learner data not captured by the Data Warehouse. These sources may include records in student information systems, timetable information, data obtained from platforms not integrated with the LMS, observations collected by instructors, or any other available data related to the students. Limiting learning analytics approaches to data found solely in enterprise data warehouses may severely reduce the capacity of instructors to use the data (West et al., 2015). These data need to be combined to offer instructors a comprehensive characterization of the learning experience for each student. It should be noted that boxes 1 and 2 in the model provide a simple categorization of the usually highly complex data topologies present in educational institutions. The internal structure and topology of these data sources may vary significantly, which is not the focus of the model.

\subsubsection{Box 3: Data Import}

The process to combine these data sources is represented in the model by the entity Data Import. It includes procedures such as data cleaning, and various transformations to generate a set of student attributes suitable for use by instructors. For example, the stream of events derived from a discussion forum (e.g., date and time each post has been written, type of contribution, text of the message, etc.) is transformed into per-student information such as number of posts per week, number of initial questions, number of answers, or number of sessions. Designing this architectural entity also requires identifying the data sources relevant and meaningful to instructors at the course level. For example, information about enrollment figures may not be as relevant as knowing the course previously taken by students. As in the case of the previous elements (boxes 1 and 2), the details of this part of the model have been abstracted, and in practice this process will be heavily influenced by the type of sources available and the amount of processing required to make it available to instructors.

\subsubsection{Box 4: Student Data Table (SDT)}

After the import stage, student data is represented in a bi-dimensional structure in which each student (e.g., Chris in Figure 1) is characterized by a set of attributes columns. The attributes contain data about student activities (e.g., number of times a formative question has been answered incorrectly, score of a multiple-choice test, previous assessments) together with information such as enrollment or demographics data. These attributes can be different for each learning environment and the values may change over time. This bi-dimensional structure was chosen for two key reasons: 1) instructors are already likely to be familiar with the data structure (e.g., a gradebook in the LMS, or a spreadsheet); and 2), it avoids instructors having to interact directly with databases. Instructors interact with this table without being exposed to the potentially complex stages represented by boxes 1, 2, and 3 in the model. 
The connection between boxes 3 and 4 assumes a non-trivial step of articulating a set of meaningful indicators of student activity from the point of view of the learning design. This stage is usually known as "sensemaking"; it requires a detailed knowledge of the relationship between the learning design elements and the data sources.

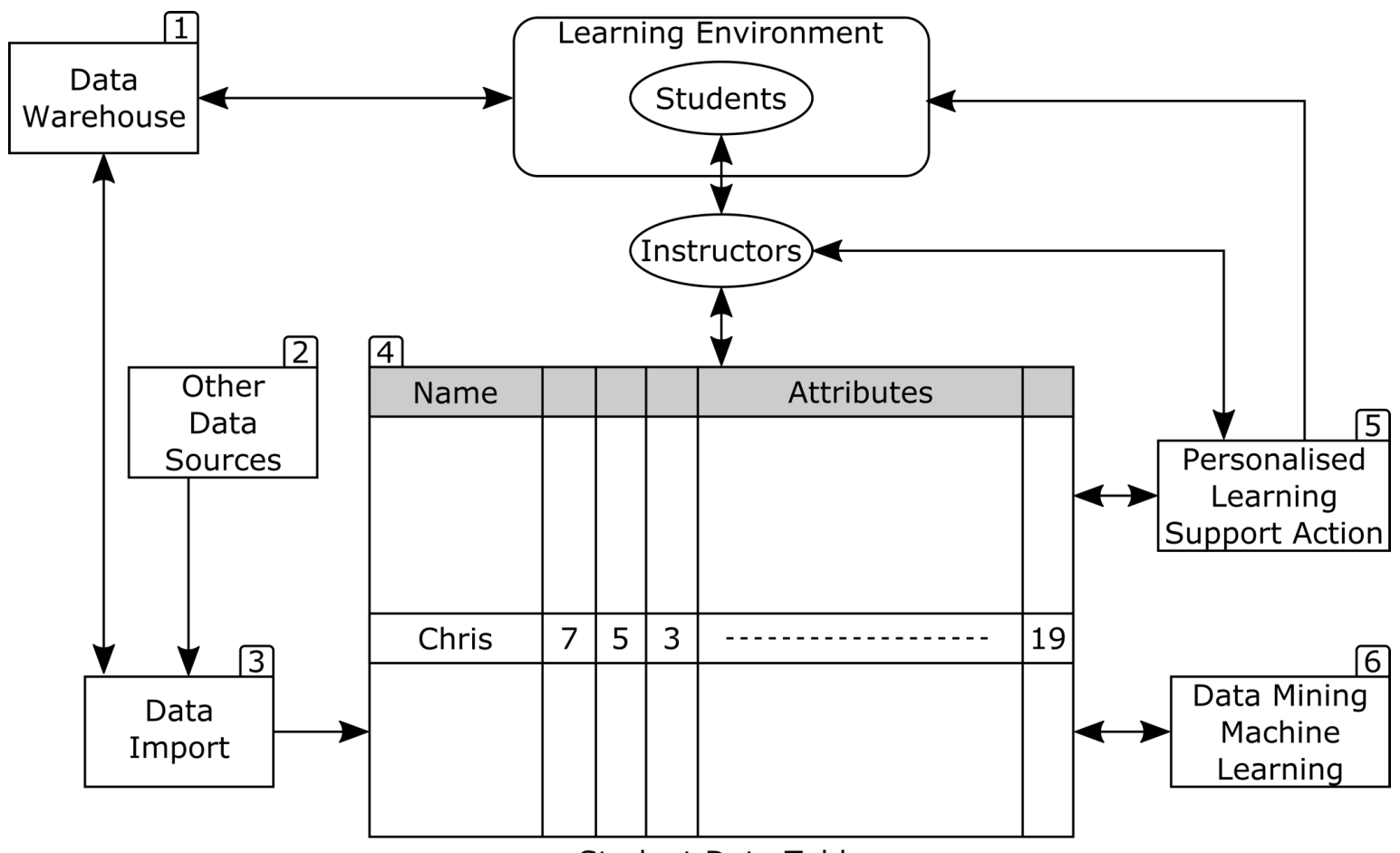

Student Data Table

Figure 3. Model for the provision of PLSAs to students.

\subsubsection{Box 5: Personalized Learning Support Actions (PLSAs)}

Instructors use the SDT to create sets of PLSAs. A PLSA contains a text statement with certain elements (words, sentences, or paragraphs) surrounded by the structure IF [CONDITION] THEN [TEXT]. Conditions are predicates defined in terms of the attributes (columns) in the table and Boolean connectors such as conjunction (AND), disjunction (OR), equality (EQUALS), negation (NOT), etc. The conditions are evaluated using the attribute values for each student and the text is included if the condition is true or ignored otherwise. PLSAs may also contain an additional condition as a filter to select or ignore the processing of certain students. Section 5.1 contains a more in-depth description of PLSAs.

The conditions and the filter in a PLSA may range from the very simplistic to the highly sophisticated. For example, a condition may detect students who just joined the course and dispatch an introductory welcome message. Another example may be a PLSA that uses a condition to identify those students taking a field trip today and sending a reminder of the aim of the trip and the proposed learning outcomes and activities. During the trip, students who did not upload a picture to the LMS as part of the learning activities are reminded to do so. A more sophisticated PLSA could identify student posts in a discussion forum that do not adequately meet the quality of work expected to promote deep engagement with the course concepts (through the execution of a text analysis algorithm in a plugin). These particular students may receive a suggestion to go beyond stating facts and then given examples of more elaborate arguments.

While the preceding exemplars target students who require some form of learning remediation, the same process can also be applied to high-performing students. A PLSA can be designed to acknowledge their progress and suggest more topics for further exploration, more advanced concepts to connect with the course material, or more advanced study techniques. Figure 3 shows an example of PLSA. The filter condition at the top selects only students who have not watched one video. There are three conditions defined depending on the attribute values for each video, and the text suggests topics if the corresponding video has not been seen.

\subsubsection{Box 6: Data Mining/Machine Learning}

The model also proposes the integration of data mining and machine learning algorithms to expand the information contained in the SDT. These algorithms typically produce a statistical model used to estimate a certain attribute in the learning environment or create a new attribute with the result of a classification scheme. For example, a clustering algorithm can be used to read the current information in the SDT and group in the same categories those students with similar attribute values. This result would be reflected in a new column with as many values as identified categories. Once this result is produced, ISSN 1929-7750 (online). The Journal of Learning Analytics works under a Creative Commons License, Attribution - NonCommercial-NoDerivs 3.0 Unported (CC BY-NC-ND 3.0) 
instructors can review the categories and use them to create a PLSA that provides different suggestions to students depending on the value of the category attribute. This interface with machine learning algorithms can also be used to incorporate statistical models computed using data from a previous cohort. The implementation of this communication or interface can be done using conventional encapsulation mechanisms through formal interfaces or class inheritance mechanisms that accommodate external elements. For example, the data from the prior edition can be used to derive a linear regression model to estimate students' midterm examination scores. The resulting regression equation is used with the data in the SDT to create a new column with the estimated score for the exam. Instructors can then use this attribute in the conditions part of a PLSA to provide personalized suggestions to students before the exam.
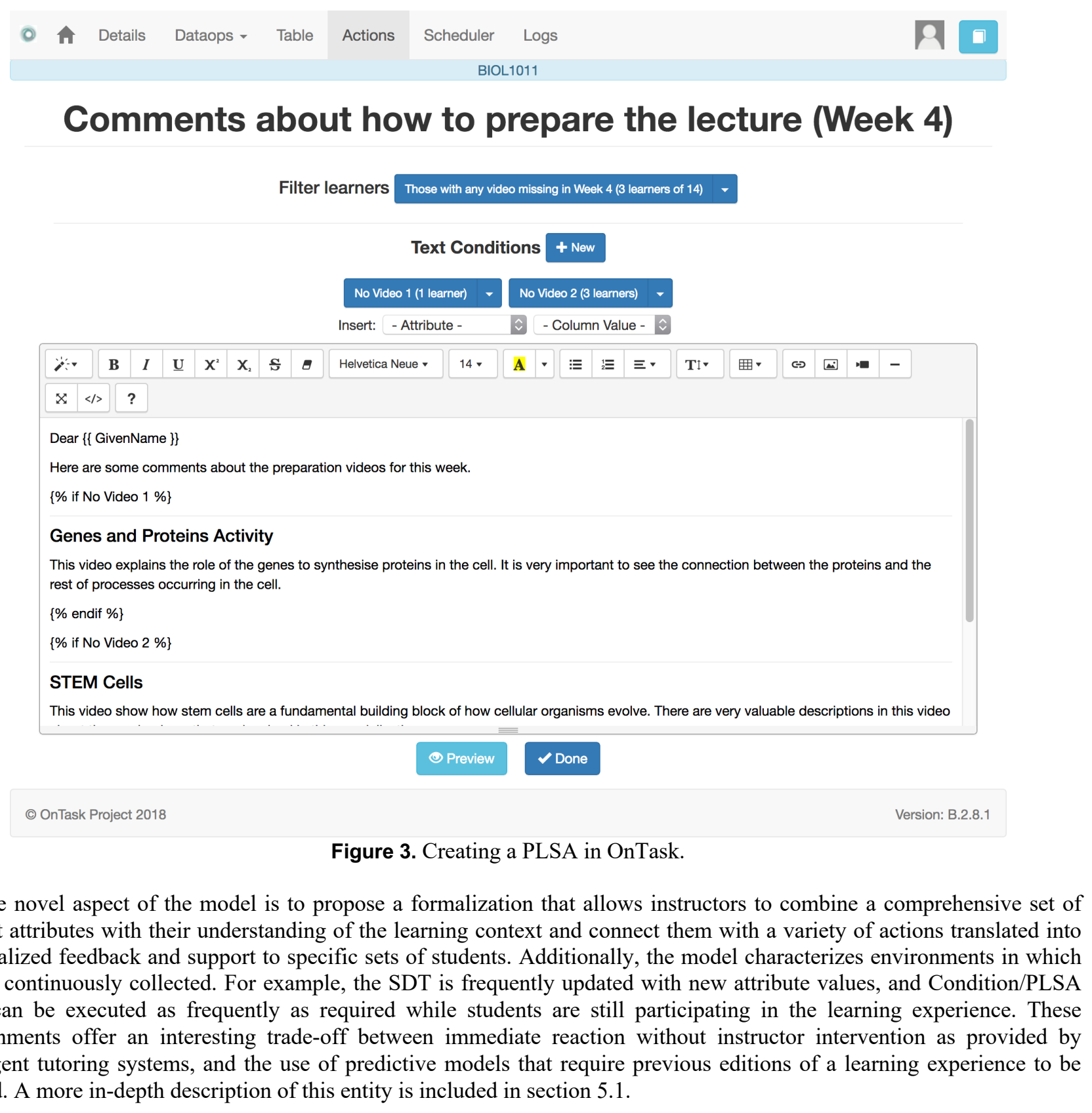

4.2. Main Stakeholders

The model considers two stakeholder groups at the centre of its structure (see Figure 2). A review of the relationships between these groups and the model follows.

\subsubsection{Students}

Similar to Laurillard's conversational framework, the model assumes an ongoing dialogue between instructor and student to better understand the concepts, theory, structure, and activities that comprise a learning experience. The interchange of 
discussions is aided by student reflection on their learning and assessment practices - both formative and summative. Hence, the conceptual model assumes that students will receive frequent, relevant, and personalized information and feedback from their instructors, the answers and reactions derived from the PLSAs are imported into the student data table, and these actions are used to prompt a dialogue between students and instructors. For example, when students complete an online task, they receive individualized feedback with suggestions specific about their overall learning process (not simply correct/incorrect message) and self-regulation effort. The feedback message can be personalized at the level of paragraphs by using the conditions written in terms of the student attributes. The reaction of students to these comments, or answers to questions specifically about this feedback can then be incorporated as the attributes used for future PLSAs. Other PLSAs may target aspects such as study strategies. The data collected may point to improvements on how students organize their study sessions. Instructors may provide different suggestions at crucial times for each student depending on his/her habits. The ultimate goal of these actions is to address the need for more comprehensive support that goes beyond performance updates (Corrin \& de Barba, 2014; McPherson, Tong, Fatt, \& Liu, 2016).

\subsubsection{Instructors}

Laurillard's framework stresses the importance for instructor adaptation of learning tasks and feedback aligned with individual student learning needs (conceptualizations). This capacity to modify learning tasks that best complement a learner's stage of development and understanding requires intimate knowledge of the course learning design — tasks and assessments. Similarly, the model recognizes that instructors are the agents most attuned to the instructional strategies and learning contexts associated with individual courses. We acknowledge that these contexts are often highly diverse and have a significant impact on how data relate to the achievement and progression of student learning outcomes (Gašević, Dawson, Rogers, \& Gasevic, 2016). By offering instructors the opportunity to manage the student data table they can better make sense of the diversity of student attributes, their connection with the implemented learning design, and, from this, specify the appropriate conditions to deploy PLSAs. This process well reflects Laurillard's concept of adaptation presented in the conversational framework. An instructor's familiarity with the learning design gives them contextual information to create personalized messages that will promote student reflection and prompt the integration of the feedback into their own conceptions. Instructors can use PLSAs to convey suggestions and reflections that go beyond a single activity and apply to the student's overall approach to learning. By placing instructors in control of delivering the PLSAs, they can also adapt the frequency and timing of the actions. They can combine actions at regular intervals (for example, once a week) with actions prompted by certain conditions satisfied by the variables in the student data table. Including the instructors as a central group in the model helps to address their need for a platform sufficiently flexible to adapt to local contexts, afford meaningful support actions, and provide a relative cost-benefit advantage over existing paradigms (Colvin et al., 2016; Macfadyen \& Dawson, 2012).

\subsubsection{Other Stakeholder Groups}

While the model emphasizes the importance of students and instructors, we do acknowledge the need to consider additional stakeholders within the institution. These added stakeholders have a significant influence on the adoption of learning analytics at the institutional level (Ferguson et al., 2014). For instance, increasingly, academic teaching staff are supported by a range of learning and teaching specialists who rely on a diverse range of learning technologies. As such these specialist areas (pedagogical and technical) have a strong influence on how data (student attributes) are captured, shared, and accessible through the system as well as aiding the translation of data into actionable insights.

Furthermore, due to the challenges that students often face with transitioning to higher education, many institutions have support staff and processes to address issues such as dropout rates, student engagement, achievement, or retention (Tinto, 2006, 2012). Such learning and teaching support typically encompass a range of services including the provision of generic learning advice (e.g., writing literacy, study skills), course advice and enrollment, career services, library services, accommodation, pastoral care, and support for equity groups. Additionally, the model, as presented, needs to be considered within its institutional context. For example, the policies around data management can vary substantially depending on the institution, or even the bodies responsible for articulating the policies around this area (for example, in the K-12 space).

Although the model has been placed in the context of student support actions, so its relation with various stakeholder groups justified, its deployment still requires the provision of an architectural view, and an implementation that can be used by an instructor in real scenarios similar to those discussed in Section 3. The following section provides a detailed specification of the architectural view and implementation of the model.

\section{Architectural View}

The model described in Section 4 has been used to derive the architecture of OnTask, a client-server, web-based software tool supporting the provision of PLSAs at scale. The architecture is illustrated in Figure 4. The following assumptions were considered in its design: 1) it must allow instructors to easily exchange their PLSAs; 2 ) the platform is part of an ecosystem of software tools supporting learning and teaching processes and as such it must provide comprehensive access to its functionality through application programming interfaces (APIs); and 3) its functionality must be easily extensible through modular blocks. The numbers in Figure 2 represent the different elements of the API, discussed further in Section 5.2.

ISSN 1929-7750 (online). The Journal of Learning Analytics works under a Creative Commons License, Attribution - NonCommercial-NoDerivs 3.0 Unported (CC BY-NC-ND 3.0) 


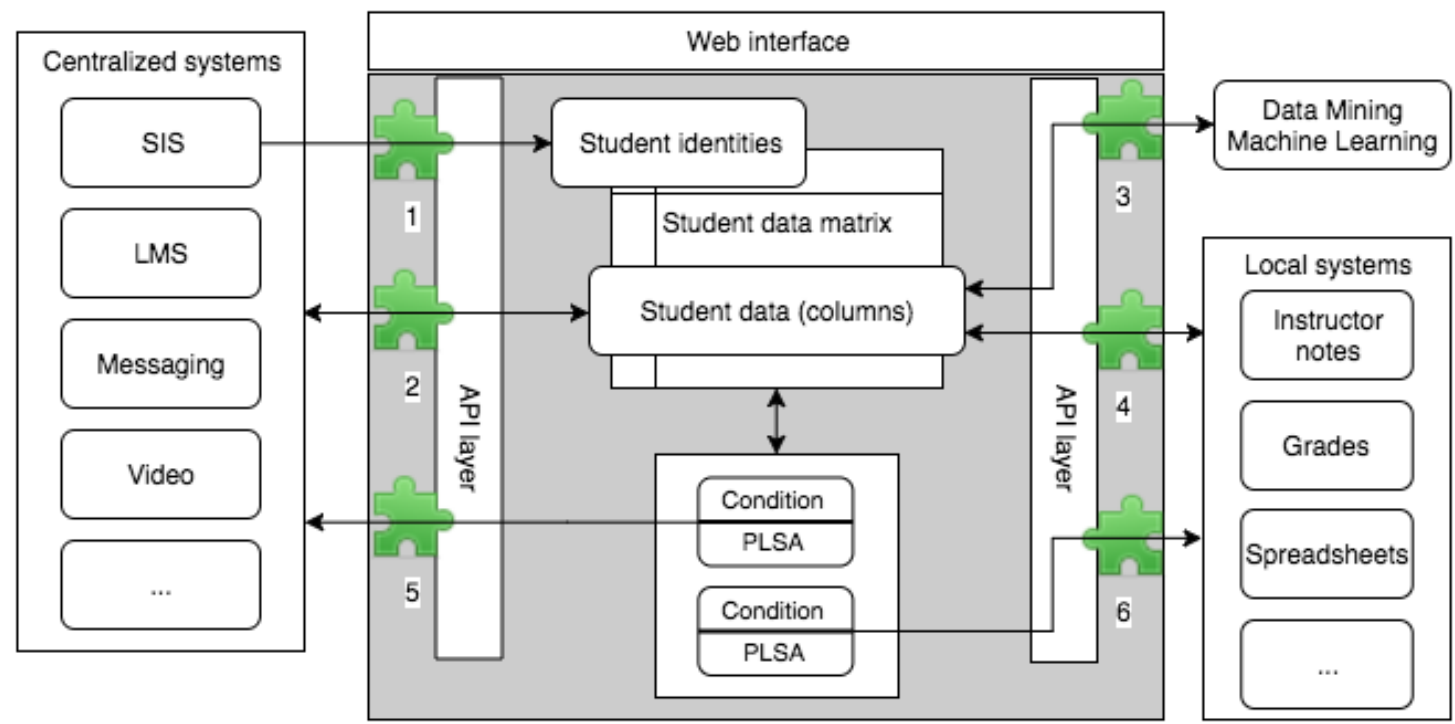

Figure 5. Architecture of the OnTask system.

The architecture maintains the SDT described in the conceptual model as its main data repository. Instructors manage the student data tables of the courses in which they are involved with columns customized for each learning context. The architecture proposes two interfaces: a conventional web interface for regular users, and a modular, extensible API to facilitate interoperability with other platforms.

\subsection{Web Interface}

In the following description of the web interface, the term "users" refers to course instructors. The functionality offered through the web interface is divided into three categories. The first contains the operations to import and export data into the platform. In a conventional workflow, instructors first upload information about the student cohort (first name, last name, student id, etc.) as the initial data in the SDT. The platform allows users to merge various data sources as additional columns in the table. These sources are assumed to have a two-dimensional structure similar to the SDT and at least one column that uniquely identifies the student in each row. When merging data from a new source, the platform allows users to specify the actions to deal with students only present in the existing table, and with students only existing in the new data source. Through the repeated use of these operations, users can construct an SDT personalized to their needs in a course and can be shared with other users within the system. At any point during the manipulation of the SDT, users may download or export its content into a format suitable for manipulation by other tools (e.g., comma-separated values). This step to build the proper SDT structure for a course can also be done in advance by support personnel familiar with the available institutional data sources and the needs from the instructor.

The second category of functionality contains regular operations to manipulate the student data table. Users may attach descriptions to columns to clarify their data, filter rows based on certain values, sort the rows based on various criteria, delete columns, allow access to the table to other users, or obtain basic summary statistics. This functionality offers basic data exploration functionality with a set of simple steps. In addition, the architecture contemplates the use of arbitrary transformations of the SDT calculated by external data mining or machine learning algorithms. For example, instructors may request an additional attribute that identifies students at risk of dropping the course. This attribute has been calculated by an external algorithm and automatically included as part of the SDT.

The third functional category contains the operations required to manage the PLSAs. Users select the type of PLSA from a pre-defined set of variants depending on the support actions. The initial actions considered in this version of the architecture include:

1. Notification sent to students through messaging applications (e.g., email and SMS).

2. Notification sent to instructors through messaging applications.

3. Data sent to other services for further processing (e.g., a CRM platform).

4. Provision of personalized content on a HTML page.

PLSAs of the above types 1 to 3 include steps to push the information proactively through other channels (sending the email or the creation of additional columns). For these actions, users can choose to execute them directly through the platform or schedule their execution at a specific time. The actions notifying users through messaging platforms or sending data to other services for further processing assume that such functionality is provided by another platform. Actions of type 4 (making a resource available at a known internet address) do not require explicit execution; instead, instructors can simply enable or disable them as needed. 
PLSAs contain conditions to select users for the action, and elements to include in a text. Users edit these conditions as a predicate containing the attribute names corresponding to the columns in the student data table connected by Boolean operators. This formalism allows users to write conditions that will target only a subset of students or to write conditions that combine data obtained from different sources. For example, an SDT may include information about engagement with videos and another column with the scores of an assignment related to the videos. The instructor may create a condition that selects those students who did not watch the video and had a score for the assignment below a certain threshold (e.g., 50\%). The text in the PLSA will be framed based on these conditions.

When the number of attributes per student is large (for example, 100 columns or observations per student), users may combine the conditions and PLSAs and create additional columns based on already existing data. For example, the SDT may contain attributes such as assessment grades, video engagement, participation in a discussion forum, attendance at lectures, etc. The user may write a condition that selects those students not attending the lectures and invoke the execution of a clustering algorithm that groups them in terms of their behaviour with respect to the other attributes. The instructor then analyzes the resulting clusters and creates additional PLSAs to provide specific resources to the members of these clusters through an HTML page (PLSA action type 4) embedded in the LMS.

\subsection{Application Programming Interface}

Although the main users of the proposed architecture are the instructor and students accessing the content derived from the PLSAs, the functionality described by the model and the architecture is closely related to information sources and processes that already exist in educational institutions and need to be properly integrated. The support for teaching and learning processes usually requires an ecosystem of tools and services with different levels of integration. The architecture needs to account for this scenario and allow for the exchange of data among the agents in this ecosystem. Additionally, the architecture should allow users to envision and explore new conditions and PLSAs connecting new data sources with instructional designs. These reasons motivated the inclusion of the API layer providing comprehensive exposure to most of the functionality. We have identified six functional categories accessible through the API, represented by the numbered pieces in Figure 4 .

\subsubsection{Import Student Identity Information}

The functionality to import student identity information (number 1 on Figure 2) is available through the API because educational institutions typically store official student records in platforms outside the LMS. The information is derived from enrollment processes executed before students participate in a learning experience. Institutions may store this information in multiple platforms or data formats. The architecture addresses this variability offering the operations through the API so that these external services may import and/or update the columns directly in the relevant SDT. An example of this feature is for an institution to create and populate the SDT for all courses at the start of the learning experience with the information extracted from the enrollment service and then update that information at regular intervals throughout the duration of the experience. The availability of this functionality through the API also allows the architecture to be extended by integrating functional blocks that support formats or services that cannot be connected directly through the API.

\subsubsection{Import/Export data}

The functionality in this category (number 2 on Figure 2) assumes the existence of an SDT and offers operations to import additional data into the table or export all or part of its content. For example, instructors may need to import information from the gradebook stored in the LMS, data extracted from an e-portfolio platform, or usage of the lecture capture system. Depending on the architecture of these existing services, a bespoke middleware layer may be needed to extract the data from the platform (e.g., the e-portfolio system) and use the proposed API to import it to an existing SDT. This functional category also offers the possibility for other systems to extract information from the SDTs.

\subsubsection{Student Data Table Transformation}

This category of the API (number 3 on Figure 2) exposes the functionality behind the PLSAs of type 3 described in Section 5.1. The interface can be used by other services to read the data in the table, apply any algorithm, and upload its result as an extra column. There are an increasing number of data processing applications offered in SaaS format (Software as a Service). The inclusion of this API category allows instructors to use these services to produce new results then used to define Condition/PLSA pairs.

\subsubsection{Import/Export student information from local systems}

This expansion point (number 4 on Figure 2) integrates functional blocks that exchange data between the SDT and systems not directly supervised by the institution. For example, instructors collect observations with a platform specific to a course that need to be incorporated into the student data table. Analogously, a course may use a platform to visualize student activity not hosted by any central system but needing to access the information in the SDT.

As in the case of the previous category, this portion of the API allows new services to provide additional data sources, such as a mobile app used to scan student ID cards to check for attendance.

\subsubsection{Actions using centralized systems}

This expansion point (number 5 on Figure 2) allows OnTask to request a service provided by the central systems leveraging ISSN 1929-7750 (online). The Journal of Learning Analytics works under a Creative Commons License, Attribution - NonCommercial-NoDerivs 3.0 Unported (CC BY-NC-ND 3.0) 
enterprise-scale capacity. For example, sending emails or SMS to students, or triggering a workflow in a CRM platform.

\subsubsection{Actions using local systems}

The last expansion point (number 6 on Figure 2) is analogous to the previous one but provides the integration of actions that require a service hosted on a non-centralized platform. Examples of this functionality would include special visualizations or personalized data made available to tutors and/or students.

In the view proposed in the architecture, the institutional information technology and business intelligence are the main units in charge of either implementing new modules and/or building (and possibly sharing through an open source community) new modules that enable data interoperability between OnTask and institution-specific centralized/local systems. This approach seeks to align the architecture with the existing organizational ecosystem of learning and teaching tools and approaches.

\subsection{Implementation}

The architecture described in this paper has been implemented as the open-source system OnTask. The platform is available for download from the Github platform ${ }^{1}$ under MIT License. Two versions of the code have been implemented using two different stacks: the Django web framework and the NodeJS framework. Both combine a web interface, a service-oriented paradigm with standardized APIs, and an ORM abstraction to connect with multiple databases (e.g., MySQL, Microsoft SQL, and PostgreSQL). The choice of these two frameworks to implement two versions was motivated by the current level of maturity, the wide variety of libraries to promote the expansion of its functionality within the learning analytics community, and the possibility for easy deployment, both in-house or cloud-hosted.

The user interface has been designed based on the experience gathered from users through versions 1 and 2 of SRES (Liu et al., 2017; McDonald et al., 2016). The objective is to provide instructors and LDS personnel with a workflow through an intuitive process with a clear connection to the learning process. Figure 1 illustrates an example of this workflow.

Figure 3 shows the screen where users create a PLSA to deliver a personalized email. The message contains a greeting personalized with the student's first name followed by a list of items included only if the condition is satisfied when evaluated with the attributes available for each student. In the example shown in Figure 3, the suggestion of the topic Genes and Proteins Activity is included in the message if the student has not seen the corresponding video. Analogously, if the second condition is true, it means the student did not watch the second video, and the suggestion about STEM Cells is included. The combination of conditions with granular comments allows for the provision of highly personalized messages to large numbers of students.

The conditions stated in terms of the attributes available for the students can be seen as a lightweight form of knowledge management that captures student support strategies that refer to specific elements of the instructional design and are adopted by instructors. With this formalism, OnTask may be used to capture the highly specialized insight that instructors usually have and its connection with support actions that can be re-executed, reused, and shared with other learning scenarios.

The software architecture supporting this paradigm in OnTask is organized in the following layers:

- Authentication and Access Management: This layer offers authentication through the OAuth API. It allows single sign-on as well as the integration of institutional authentication platforms. The layer also includes control lists to differentiate the level of access to each SDT and operations.

- Data management REST API: This layer allows other applications to connect directly to the database to manage the internal data structures and expose all the import/export functionality. The same API is used within the system to support the operations through the web interface.

- SDT builder: This layer implements the functions that allow users to select data sources and perform transformations such as joining tables, aggregating data from different columns, etc.

- PLSAs builder: This layer includes the functionality to create the actions (either manually or with a wizard) and attach them to the required PLSAs.

- Notification Gateway REST API: This layer contains the functionality to handle most of the computation and communication required by the PLSAs; namely, sending messages, serving the HTML reports, or transferring the information to third-party services (e.g., CRM systems, social networks, etc.)

The structure of these layers promotes the expansion of the platform in three aspects:

- The creation of bespoke plugins to allow the platform to be tightly integrated with already existing institutional services. These plugins can expand the OnTask functionality and at the same time increase the operations visible through the categories described in Section 5.2. The possibility to deploy these plugins is especially important to accommodate the high variation with respect to data sources available to instructors or to increase the available support actions.

- The creation of middleware to integrate OnTask loosely with additional institutional systems. This type of expansion requires no changes in the internal structure of the tool, but instead external agents need to be created to invoke the REST API and perform the required operations.

- Finally, the tool offers comprehensive import/export functions to promote the exchange of artefacts related to the workflows among instructors and LDS personnel.

\footnotetext{
${ }^{1} \mathrm{https}: / /$ github.com/abelardopardo/ontask b and https://github.com/argsen/ontask

ISSN 1929-7750 (online). The Journal of Learning Analytics works under a Creative Commons License, Attribution - NonCommercial-NoDerivs 3.0 Unported (CC BY-NC-ND 3.0)
} 


\section{Open Source Learning Analytics}

Learning analytics functionality is increasingly offered by vendors ranging from existing LMS and ERP (Enterprise Resource Planning) providers to other providers specializing in this area. For faculty and university leaders, questions about access to algorithms that underpin the analytics models and feedback outputs become pedagogical and privacy issues. Previous proposals for Open Learning Analytics (Siemens et al., 2011) have called for greater recognition for the critical need for openness in analytics in education. Recent authors have confirmed the potential for open learning analytics models (Chatti et al., 2014; Muslim, Chatti, Bashir, Barrios Varela, \& Schroeder, 2018; Schroeder, Mughal, Chatti, \& Muslim, 2017) to deal with the increasing complexity emerging in learning environments. However, the area still faces multiple challenges to provide a concrete implementation of these models due to its wide scope, and remain mostly focused on analytics tasks and not on how to best support students and instructors in a learning scenario (Chatti et al., 2017; Cooper, 2014). The initiative described in this paper seeks to provide a concrete conceptual and development plan to offer a tool and methodology to foster the exchange of processes, data, and models among researchers and practitioners.

Challenges for the adoption of an open learning platform are largely due to the breadth of the area and the numerous aspects to address. Varying configurations of LMS, social media, and enterprise systems in different universities present problems in creating an integrated open system. Rather than addressing the problem as a whole, OnTask focuses on supporting students while they participate in a specific learning experience. Single functionality software, such as OnTask, benefit from, and help to advance, open learning analytics by utilizing an open framework. The design of OnTask aims to support instructors in formulating their insights about the learning environment as a connection between data and student support actions. As designed, the platform ensures compatibility with current initiatives such as xAPI, Caliper, and the Open Learning Analytics Initiative (led by the Apereo Foundation together with other institutions). We position OnTask in the area of actions with respect to the existing tool ecosystem as it focuses in the provision of student support by capturing the insight of instructors at the course level.

Learning is highly sensitive to the instructional context; what works for one institution in one context may not work for another. Even so, there is a large potential for sharing not only the data, but also the conditions in which the data are being used for PLSAs. Based on the experience with previous versions of the described conceptual model (SRES versions 1 and 2), we envision OnTask as the conduit needed to share all aspects of the process to deploy PLSAs. Building on the currently emerging set of open standards in learning analytics, OnTask will contribute to broadening recognition of OLA in general and will enable integration in multiple institutional settings.

\section{Conclusions}

Learning analytics needs to widen its focus to explore the use of data to provide more general student support actions. This paper outlined a student and instructor centred conceptual model and an architecture to aid instructors to specify and deploy data-supported, personalized, scalable student support processes. The model espoused seeks to integrate the use of comprehensive data sources, instructor knowledge of the learning context, and a formal description of the connection between actions and personalized learning support actions (PLSAs).

A key component of the model resides in its capacity to capture the input from the instructor with a lightweight formalism. Only through this hybrid process we can fully address the complexity of the learning context, namely the diversity of teaching approaches, student cohorts, assessment and design methods, and programmatic curriculum pathways. The model uses a simple representation of student data close to conventional tools such as spreadsheets with the hope of lowering the adoption barrier for instructors and LDS personnel (Obrenovic \& Gašević, 2008). The ability for instructors to create their own condition/s not only aids the personalization of the PSLAs but also provides a more personal, relevant dimension to facilitate instructor uptake. The tool described in this paper captures this expertise and opens the possibilities for the deployment of PLSAs. Although there are situations in learning environments too complex to describe with the proposed condition/PLSA structures, the tool offers a trade-off between complexity and feasibility that has not been properly explored. The tool is available under an opensource license to promote the contribution of functionality and exchange of artefacts among the community of researchers and practitioners.

The adoption of such a tool is heavily dependent on how to support instructors throughout the different stages of the process. For example, data management is an area that can be provided to personnel with the right set of skills to produce and populate the data table with the relevant student information connected to the learning design. Instructional designers can also envision common rule patterns that apply to certain design elements, provided to instructors to frame the personalized messages. We envision this multipronged support as an essential element to promote institutional adoption of this approach.

Future steps for this initiative include the deployment of several pilots to support the ongoing provision of support actions, widen the engagement to courses with large or highly diverse student cohorts, gather user feedback about the aspects of the tool that need to be expanded, and explore the factors required to promote its adoption at the institutional level. 


\section{Declaration of Conflicting Interest}

The author(s) declared no potential conflicts of interest with respect to the research, authorship, and/or publication of this article.

\section{Funding}

Support for this research has been provided by the Australian Government Office for Learning and Teaching.

\section{Acknowledgements}

The views expressed in this article do not necessarily reflect the views of the Australian Government Office for Learning and Teaching. The authors would like to acknowledge the contribution of Dr. Jenny McDonald (University of Auckland) to the design and implementation of this platform as well as for several discussions about the strategic view driving them.

\section{References}

Baker, R. S. (2016). Stupid tutoring systems, intelligent humans. International Journal of Artificial Intelligence in Education, 26(2), 600-614. http://dx.doi.org/10.1007/s40593-016-0105-0

Brooks, C., Greer, J., \& Gutwin, C. (2014). The data-assisted approach to building intelligent technology-enhanced learning environments. In J. A. Larusson \& B. White (Eds.), Learning analytics: From research to practice (pp. 123-156). New York: Springer. http://dx.doi.org/10.1007/978-1-4614-3305-7 7

Buckingham Shum, S., Knight, S., McNamara, D., Allen, L., Bektik, D., \& Crossley, S. (2016). Critical perspectives on writing analytics. Proceedings of the $6^{\text {th }}$ International Conference on Learning Analytics and Knowledge (LAK '16), 25-29 April 2016, Edinburgh, UK (pp. 481-483). New York: ACM. http://dx.doi.org/10.1145/2883851.2883854

Bull, S., \& Kay, J. (2016). SMILI $\odot:$ : A framework for interfaces to learning data in open learner models, learning analytics and related fields. International Journal of Artificial Intelligence in Education, 26(1), 293-331. http://dx.doi.org/10.1007/s40593-015-0090-8

Chatti, M. A., Lukarov, V., Thüs, H., Muslim, A., Yousef, A. M. F., Wahid, U., . . Schroeder, U. (2014). Learning analytics: Challenges and future research directions. eleed, 10(1).

Chatti, M. A., Muslim, A., \& Schroeder, U. (2017). Toward an open learning analytics ecosystem. In B. K. Daniel (Ed.), Big data and learning analytics in higher education (pp. 195-219). Switzerland: Springer. http://dx.doi.org/10.1007/9783-319-06520-5 12

Clow, D. (2012). The learning analytics cycle: Closing the loop effectively. Proceedings of the $2^{\text {nd }}$ International Conference on Learning Analytics and Knowledge (LAK '12), 29 April-2 May 2012, Vancouver, BC, Canada (pp. 134-138). New York: ACM. http://dx.doi.org/10.1145/2330601.2330636

Colvin, C., Rogers, T., Wade, A., Dawson, S., Gašević, D., Buckingham Shum, S., . . Fisher, J. (2016). Student retention and learning analytics: A snapshot of Australian practices and a framework for advancement. Caberra, ACT: Australian Government Office for Learning and Teaching.

Cooper, A. (2014). Learning analytics interoperability: The big picture in brief. Learning Analytics Community Exchange, March 26, 2014. http://www.laceproject.eu/blog/learning-analytics-interoperability-briefing

Corrin, L., \& de Barba, P. (2014). Exploring students' interpretation of feedback delivered through learning analytics dashboards. Proceedings of the $31^{\text {st }}$ Annual Conference of the Australasian Society for Computers in Learning in Tertiary Education (ASCILITE 2014), 23-26 November 2014, Dunedin, New Zealand (pp. 629-633). Australasian Society for Computers in Learning in Tertiary Education.

Corrin, L., \& de Barba, P. (2015). How do students interpret feedback delivered via dashboards? Proceedings of the $5^{\text {th }}$ International Conference on Learning Analytics and Knowledge (LAK '15), 16-20 March 2015, Poughkeepsie, NY, USA (pp. 430-431). New York: ACM. http://dx.doi.org/10.1145/2723576.2723662

Crossley, S. A., Kyle, K., \& McNamara, D. S. (2015). The tool for the automatic analysis of text cohesion (TAACO): Automatic assessment of local, global, and text cohesion. Behavior Research Methods, 48: 1227, 1-11. http://dx.doi.org/10.3758/s13428-015-0651-7

Echeverria, V., Martinez-Maldonado, R., Granda, R., Chiluiza, K., Conati, C., \& Buckingham Shum, S. (2018). Driving data storytelling from learning design. Proceedings of the $8^{\text {th }}$ International Conference on Learning Analytics and Knowledge (LAK '18), 5-9 March 2018, Sydney, NSW, Australia (pp. 131-140). New York: ACM. http://dx.doi.org/10.1145/3170358.3170380

Evans, C. (2013). Making sense of assessment feedback in higher education. Review of Educational Research, 83(1), 70120. http://dx.doi.org/10.3102/0034654312474350

Experience API. (2016). Github repository. Retrieved from https://github.com/adlnet/-xAPI-Spec/blob/master/xAPI.md

Ferguson, R., Macfadyen, L., Clow, D., Tynan, B., Alexander, S., \& Dawson, S. (2014). Setting learning analytics in context: Overcoming the barriers to large-scale adoption. Journal of Learning Analytics, 1(3), 120-144. http://dx.doi.org/10.1145/2567574.2567592

Gašević, D., Dawson, S., Rogers, T., \& Gasevic, D. (2016). Learning analytics should not promote one size fits all: The 
effects of instructional conditions in predicting academic success. The Internet and Higher Education, 28, 68-84. http://dx.doi.org/10.1016/j.iheduc.2015.10.002

Gašević, D., Dawson, S., \& Siemens, G. (2015). Let's not forget: Learning analytics are about learning. TechTrends, 59(1), 64-75. http://dx.doi.org/10.1007/s11528-014-0822-x

Hattie, J., \& Timperley, H. (2007). The power of feedback. Review of Educational Research, 77(1), 81-112. http://dx.doi.org/10.3102/003465430298487

Hegazi, M. O., \& Abugroon, M. A. (2016). The state of the art on educational data mining in higher education. International Journal of Computer Trends and Technology, 31, 46-56.

IMS Consortium. (2016). Caliper analytics. Retrieved from http://www.imsglobal.org/activity/caliperram

Jayaprakash, S. M., Moody, E. W., Eitel, J. M., Regan, J. R., \& Baron, J. D. (2014). Early alert of academically at-risk students: An open source analytics initiative. Journal of Learning Analytics, 1(1), 6-47.

Kahn, I., \& Pardo, A. (2016). Data2U: Scalable real time student feedback in active learning environments. Proceedings of the $6^{\text {th }}$ International Conference on Learning Analytics and Knowledge (LAK '16), 25-29 April 2016, Edinburgh, UK (pp. 249-253). New York: ACM. http://dx.doi.org/10.1145/2883851.2883911

Laurillard, D. (2013). Rethinking university teaching: A conversational framework for the effective use of learning technologies (2nd ed.). London: Routledge.

Lewkow, N., Zimmerman, N., Riedesel, M., \& Essa, A. (2015). Learning analytics platform: Towards an open scalable streaming solution for education. In O. C. Santos et al. (Eds.), Proceedings of the $8^{\text {th }}$ International Conference on Educational Data Mining (EDM2015), 26-29 June 2015, Madrid, Spain (pp. 460-463). International Educational Data Mining Society.

Liu, D. Y. T., Bartimote-Aufflick, K., Pardo, A., \& Bridgeman, A. J. (2017). Data-driven personalization of student learning support in higher education. In A. Peña-Ayala (Ed.), Learning analytics: Fundaments, applications, and trends: A view of the current state of the art: Springer. http://dx.doi.org/10.1007/978-3-319-52977-6 5

Liu, R., Patel, R., \& Koedinger, K. R. (2016). Modeling common misconceptions in learning process data. Proceedings of the $6^{\text {th }}$ International Conference on Learning Analytics and Knowledge (LAK '16), 25-29 April 2016, Edinburgh, UK (pp. 369-377). New York: ACM. http://dx.doi.org/10.1145/2883851.2883967

Lonn, S., Aguilar, S. J., \& Teasley, S. D. (2015). Investigating student motivation in the context of a learning analytics intervention during a summer bridge program. Computers in Human Behavior, 47, 90-97. http://dx.doi.org/10.1016/j.chb.2014.07.013

Ma, W., Adesope, O. O., Nesbit, J. C., \& Liu, Q. (2014). Intelligent tutoring systems and learning outcomes: A metaanalysis. Journal of Educational Psychology, 106(4), 901-918. http://dx.doi.org/10.1037/a0037123

Macfadyen, L. P., \& Dawson, S. (2012). Numbers are not enough: Why e-learning analytics failed to inform an institutional strategic plan. Journal of Educational Technology \& Society, 15(3), 149-163.

McDonald, J., Liu, D. Y. T., Moskal, A., Zeng, R., Blumenstein, M., Gunn, C., . . Pardo, A. (2016). Cross-institutional collaboration to support student engagement: SRES version 2. In S. Barker, S. Dawson, A. Pardo, \& C. Colvin (Eds.), Show Me The Learning: Proceedings of the 33rd Annual Conference of the Australasian Society for Computers in Learning in Tertiary Education (ASCILITE 2016). 28-30 November 2016, Wellington, New Zealand (pp. 397-405). Australasian Society for Computers in Learning in Tertiary Education.

McPherson, J., Tong, H. L., Fatt, S. J., \& Liu, D. Y. T. (2016). Student perspectives on data provision and use: Starting to unpack disciplinary differences. Proceedings of the $6^{\text {th }}$ International Conference on Learning Analytics and Knowledge (LAK '16), 25-29 April 2016, Edinburgh, UK (pp. 158-167 ). New York: ACM. http://dx.doi.org/10.1145/2883851.2883945

Muslim, A., Chatti, M. A., Bashir, M. B., Barrios Varela, O. E., \& Schroeder, U. (2018). A modular and extensible framework for open learning analytics. Journal of Learning Analytics, 5(1), 92-100. http://dx.doi.org/10.18608/jla.2018.51.7

Nicol, D. (2010). From monologue to dialogue: Improving written feedback processes in mass higher education. Assessment \& Evaluation in Higher Education, 35(5), 501-517. http://dx.doi.org/10.1080/02602931003786559

Nicol, D., Thomson, A., \& Breslin, C. (2013). Rethinking feedback practices in higher education: A peer review perspective. Assessment \& Evaluation in Higher Education, 39(1), 102-122. http://dx.doi.org/10.1080/02602938.2013.795518

Niculescu, C. (2016). Intelligent tutoring systems: Trends on design, development and deployment. Proceedings of the $12^{\text {th }}$ International Scientific Conference "eLearning and Software for Education” (eLSE 2016), 21-22 April 2016, Bucharest, Romania (vol. 3, pp. 280-285). Carol I National Defence University Publishing House, Bucharest, Romania. http://dx.doi.org/10.12753/2066-026X-16-218

Nye, B. D. (2014). Intelligent tutoring systems by and for the developing world: A review of trends and approaches for educational technology in a global context. International Journal of Artificial Intelligence in Education, 25(2), 177 203. http://dx.doi.org/10.1007/s40593-014-0028-6

Obrenovic, Z., \& Gašević, D. (2008). End-user service computing: Spreadsheets as a service composition tool. IEEE Transactions on Services Computing, 1(4), 229-242. http://dx.doi.org10.1109/tsc.2008.16

Pardo, A., Jovanović, J., Dawson, S., Gašević, D., \& Mirriahi, N. (2018). Using learning analytics to scale the provision of personalised feedback. British Journal of Educational Technology. http://dx.doi.org/10.1111/bjet.12592

ISSN 1929-7750 (online). The Journal of Learning Analytics works under a Creative Commons License, Attribution - NonCommercial-NoDerivs 3.0 Unported (CC BY-NC-ND 3.0) 
Pelánek, R., Rihák, J., \& Papoušek, J. (2016). Impact of data collection on interpretation and evaluation of student models. Proceedings of the $6^{\text {th }}$ International Conference on Learning Analytics and Knowledge (LAK '16), 25-29 April 2016, Edinburgh, UK (pp. 40-47). New York: ACM. http://dx.doi.org/10.1145/2883851.2883868

Romero, C., \& Ventura, S. (2013). Data mining in education. Wiley Interdisciplinary Reviews: Data Mining and Knowledge Discovery, 3(1), 12-27. http://dx.doi.org/10.1002/widm.1075

Romero Zaldívar, V. A., Pardo, A., Burgos, D., \& Delgado Kloos, C. (2012). Monitoring student progress using virtual appliances: A case study. Computers \& Education, 58(4), 1058-1067. http://dx.doi.org/10.1016/j.compedu.2011.12.003

Scaffidi, C., Shaw, M., \& Myers, B. (2005). Estimating the number of end users and end user programmers. Proceedings of the 2005 IEEE Symposium on Visual Languages and Human-Centric Computing (VL/HCC'05), 20-24 September 2005, Dallas, TX, USA (pp. 1-8). Los Alamitos, CA: IEEE Computer Society. http://dx.doi.org/10.1109/VLHCC.2005.34

Schroeder, U., Mughal, M., Chatti, M. A., \& Muslim, A. (2017). The goal-question-indicator approach for personalized learning analytics. Proceedings of the $9^{\text {th }}$ International Conference on Computer Supported Education (CSEDU 2017) 21-23 April 2017, Porto, Portugal (Vol. 1, pp. 371-378). ScitePress. http://dx.doi.org/10.5220/0006319803710378

Sclater, N., Peasgood, A., \& Mullan, J. (2016). Learning analytics in higher education: A review of UK and international practice. United Kingdom: Joint Information Systems Committee (JISC).

Shacklock, X. (2016). From bricks to clicks: The potential of data and analytics in higher education. London, UK: Higher Education Commission.

Shamah, D., \& Ohlsen, S. (2013). Student success plan: Constructing an evidence-based student support system that promotes college completion. Portland, OR: Gateway to College National Network.

Siemens, G., Gašević, D., Haythornthwaite, C., Dawson, S., Buckingham Shum, S., Ferguson, R., . . Baker, R. (2011). Open learning analytics: An integrated and modularized platform. Society for Learning Analytics and Research. http://www.elearnspace.org/blog/wp-content/uploads/2016/02/ProposalLearningAnalyticsModel SoLAR.pdf

Tinto, V. (2006). Research and practice of student retention: What next? Journal of College Student Retention, 8(1), 1-19. http://dx.doi.org/10.2190/4YNU-4TMB-22DJ-AN4W

Tinto, V. (2012). Completing college: Rethinking institutional action. Chicago, IL: University of Chicago Press.

Verbert, K., Duval, E., Klerkx, J., Govaerts, S., \& Santos, J. L. (2013). Learning analytics dashboard applications. American Behavioral Scientist, 57(10), 1500-1509. http://dx.doi.org/10.1177/0002764213479363

West, D., Huijser, H., Lizzio, A., Toohey, D., Miles, C., Searle, B., \& Bronnimann, J. (2015). Learning analytics: Assisting universities with student retention, final report (Part 1). Australian Government Office for Learning and Teaching.

Wise, A. F. (2014). Designing pedagogical interventions to support student use of learning analytics. Proceedings of the $4^{\text {th }}$ International Conference on Learning Analytics and Knowledge (LAK '14), 24-28 March 2014, Indianapolis, IN, USA (pp. 203-211). New York: ACM. http://dx.doi.org/10.1145/2567574.2567588

Wise, A. F., Vytasek, J. M., Hausknecht, S., \& Zhao, Y. (2016). Developing learning analytics design knowledge in the "middle space": The student tuning model and align design framework for learning analytics use. Online Learning Journal, 20(2), 155-182.

Wright, M. C., McKay, T., Hershock, C., Miller, K., \& Tritz, J. (2014). Better than expected: Using learning analytics to promote student success in gateway science. Change: The Magazine of Higher Learning, 46(1), 28-34. http://dx.doi.org/10.1080/00091383.2014.867209 